This document is confidential and is proprietary to the American Chemical Society and its authors. Do not copy or disclose without written permission. If you have received this item in error, notify the sender and delete all copies.

\title{
Exploring the high-temperature frontier in molecular nanomagnets: from lanthanides to actinides
}

\begin{tabular}{|r|l|}
\hline Journal: & Inorganic Chemistry \\
\hline Manuscript ID & ic-2019-01610f.R2 \\
\hline Danuscript Type: & Forum Article \\
\hline Author: & 28-Aug-2019 \\
\hline & $\begin{array}{l}\text { Complete List of Authors: } \\
\text { Maldoví, José; Max-Planck-Institut fur Struktur und Dynamik der Materie, } \\
\text { Gaita-Ariño, Alejandro; Universidad de Valencia, Instituto de Ciencia } \\
\text { Molecular (ICMol) } \\
\text { Coronado, Eugenio; Universidad de Valencia, Instituto de Ciencia } \\
\text { Molecular }\end{array}$ \\
\hline
\end{tabular}

\section{SCHOLARONE \\ Manuscripts}




\section{Introduction}

The possibility of storing binary information into a single magnetic molecule is a dream that has revolutionized the field of molecular magnetism since the early 1990s. The pursuit of minimalistic magnets exhibiting magnetic hysteresis from purely molecular origin started with the discovery of slow relaxation of the magnetization and macroscopic quantum tunneling of the magnetization in $\left[\mathrm{Mn}_{12} \mathrm{O}_{12}\left(\mathrm{O}_{2} \mathrm{CMe}\right)_{16}\left(\mathrm{H}_{2} \mathrm{O}\right)_{4}\right] \cdot 2 \mathrm{MeCO}_{2} \mathrm{H} \cdot 4 \mathrm{H}_{2} \mathrm{O} \quad\left(\mathrm{Mn}_{12} \mathrm{ac}\right),{ }^{1,2}$ popularly known as the drosophila of the single-molecule

magnets (SMMs). ${ }^{3}$ This class of magnetic entities display magnetic bistability generated by an energy barrier to the magnetization reversal. Because of their extraordinarily rich physical behavior, SMMs have been considered as ideal laboratories to study new quantum phenomena, ${ }^{4}$ such as quantum tunneling of the magnetization, while they are also potential candidates for a set of stimulating applications. ${ }^{5,6,7,8}$ The main figure of merit that evaluates the performance of an SMM is the blocking temperature $\left(T_{B}\right)$, which can be described as the highest temperature at which these nanomagnets can retain its magnetization for a given time interval. The enhancement of $T_{B}$ has then been the main target for researchers aiming at obtaining SMMs exhibiting magnetic hysteresis at high temperature.

The first generation of SMMs was based on magnetic transition metal clusters, in which an anisotropic high-spin ground state can be stabilized by superexchange interactions between a number of anisotropic magnetic centers. Despite the great attention that this kind of nano-objects attracted, the progress with regard to their energy barrier $\left(U_{\text {eff }}\right)$ and $T_{B}$ was pretty modest because of the intrinsic limitations to increase the height of the anisotropy barrier. ${ }^{9}$ Among this wide variety of transition metal clusters, the two recordbearing families are the $\left\{\mathrm{Mn}^{\mathrm{III}}{ }_{8} \mathrm{Mn}^{\mathrm{IV}}{ }_{4}\right\}$ SMMs, derived from the original $\mathrm{Mn}_{12} \mathrm{ac}$, which present $U_{\text {eff }}$ values as large as 74 $\mathrm{K},{ }^{10}$ and the $\left\{\mathrm{Mn}^{\mathrm{III}}{ }_{6}\right\}$-oximato clusters that possess practically the same record energy barrier with $\left[\mathrm{Co}_{4}(\mu\right.$ $\left.\left.\mathrm{NP}^{\mathrm{t}} \mathrm{Bu}_{3}\right)_{4}\right]\left[\mathrm{B}\left(\mathrm{C}_{6} \mathrm{~F}_{5}\right)_{4}\right]$ (where ${ }^{\mathrm{t}} \mathrm{Bu}=$ tert-butyl), being $86 \mathrm{~K}$ for $\left\{\mathrm{Mn}_{6}\right\}^{11}$ and $87 \mathrm{~K}$ for $\left\{\mathrm{Co}_{4}\right\}^{12}$.

In 2003, a novel framework to design SMMs was introduced by Ishikawa and co-workers. ${ }^{13}$ This resulted in the development of even smaller nanomagnets based on coordination compounds with a single lanthanoid ion as the source of magnetic anisotropy, which arise from the combination of unquenched spin-orbit and ligand field splitting. ${ }^{14}$ The first example of this second generation of SMMs relies on a terbium cation sandwiched by two phthalocyaninato anions. This system represented an important increase of the energy barrier $\left(U_{\text {eff }}=330 \mathrm{~K}\right)$, but 
its blocking temperature was merely $1.7 \mathrm{~K}$. Since then, a vast number of mononuclear SMMs, also known as single-ion magnets (SIMs), have been reported. ${ }^{15}$

The discovery of single-ion magnetism based on lanthanides stimulated the quest for this behavior in other types of magnetic ions in the periodic table. Still, less effort has been put so far into this direction compared with the impressive expansion of the lanthanoid-based family, and thus a considerable smaller number of examples do exist. As far as transition metal based SIMs are concerned, dozens of examples have been published, ${ }^{16}$ and while cobalt and iron are the most common magnetic ions, examples also include chromium, manganese, nickel, copper and rhenium. ${ }^{17}$ Despite these synthetic efforts, the hysteresis record in standard conditions for transition metal mononuclear SMMs is still the one of the first reported example, namely $6.5 \mathrm{~K}$ for $\left[\mathrm{K}(\right.$ crypt-222) $]\left[\mathrm{Fe}\left(\mathrm{C}\left(\mathrm{SiMe}_{3}\right)_{3}\right)_{2}\right]$, which displays an energy barrier of $226 \mathrm{~cm}^{-1} .{ }^{18}$ Also, note that magnetic hysteresis with coercivity was measured for $[(\mathrm{SIPr}) \mathrm{Co}=\mathrm{NAr}] \quad(\mathrm{SIPr} \quad=\quad 1,3$-bis $(2,6-$ diisopropylphenyl)imidazolin-2-ylidene, and $\mathrm{Ar}=2,6$ dimesitylphenyl) up to $9.5 \mathrm{~K}$ using a fast field sweep rate of $700 \mathrm{Oe} \cdot \mathrm{s}^{-1} \cdot{ }^{19}$ Another case that deserves a special mention, due to the combined theoretical and experimental effort that has been carried out, is $\mathrm{Co}\left(\mathrm{C}\left(\mathrm{SiMe}_{2} \mathrm{ONaph}\right)_{3}\right)_{2}$, where $\mathrm{Naph}$ = naphthyl group. In this SIM, which exhibits magnetic hysteresis up to $5 \mathrm{~K}$, the three vibrations that are strongly coupled to spin states have recently been identified using variable-field FIR spectroscopy, which also allowed to obtain some of the parameters that estimate the weight of the different contributions to relaxation. ${ }^{20}$ In actinides, both the number and the variety of examples are remarkable smaller compared to the case of transition metals. Practically all of the studied systems are based on uranium, ${ }^{21,22,23,24}$ with only a couple of examples based on neptunium ${ }^{25}$ and plutonium. The best reported case is the uranium complex $\left[\mathrm{U}\left(\mathrm{TpMe}_{2}\right)_{2}\right] \mathrm{I}$ with hysteresis below $5 \mathrm{~K}^{26}$

Owing to the broader number of available SIMs based on the lanthanoid-based family and to the accumulation of knowledge derived from the theoretical studies, improved design strategies have been developed to ameliorate their properties. A key example that illustrates this point is provided by the cyclooctatetraenyl metallocene complexes, where a wise choice of the lanthanoid has allowed to maximize the magnetic anisotropy, achieving blocking temperatures as high as $14 \mathrm{~K}$ in the $\mathrm{Er}^{3+}$ derivatives. ${ }^{27}$ This strategy has subsequently been improved using more compact $\pi$-clouds and thus a stronger axial magnetic field, which has resulted in a dramatic ramp-up of $T_{B}$ since 2017,28, ${ }^{29}$ with the chemical design of SIMs reaching magnetic hysteresis above liquid-nitrogen temperatures (see Fig. 1). ${ }^{30}$ Up to now, the most promising systems have been doubledecker systems based on rigid aromatic rings. This kind of coordination environment, which depend on the selected rings, favors an axially-elongated ligand field that encapsulates a lanthanide ion with an oblate $f$-shell cloud, e.g. $\mathrm{Dy}^{3+}$, which minimizes electrostatic repulsion. ${ }^{31}$ By a careful tailoring of the substituents in this type of aromatic ligands, one can envision further improvements beyond the current performances of these magnetic entities that are nowadays the high-temperature frontier of SIMs.

\section{Modeling the electronic structure in f-block single- ion magnets}

Despite this encouraging progress, modeling the properties of $f$-block molecular nanomagnets from accurate magneto-structural correlations is considered a great challenge that has awakened a remarkable interest in the field. Nowadays, there are several complementary methodologies that have been progressing in parallel with respect to the experimental discoveries. There is an upward trend in molecular magnetism to standardize on methods based on the chemical structure, either electrostatic or, more commonly, $a b$ initio. In contrast, spectroscopists studying the optical transitions of the rare earths tend to rely on phenomenological crystal field methods, fitting a large range of experimental energy levels.

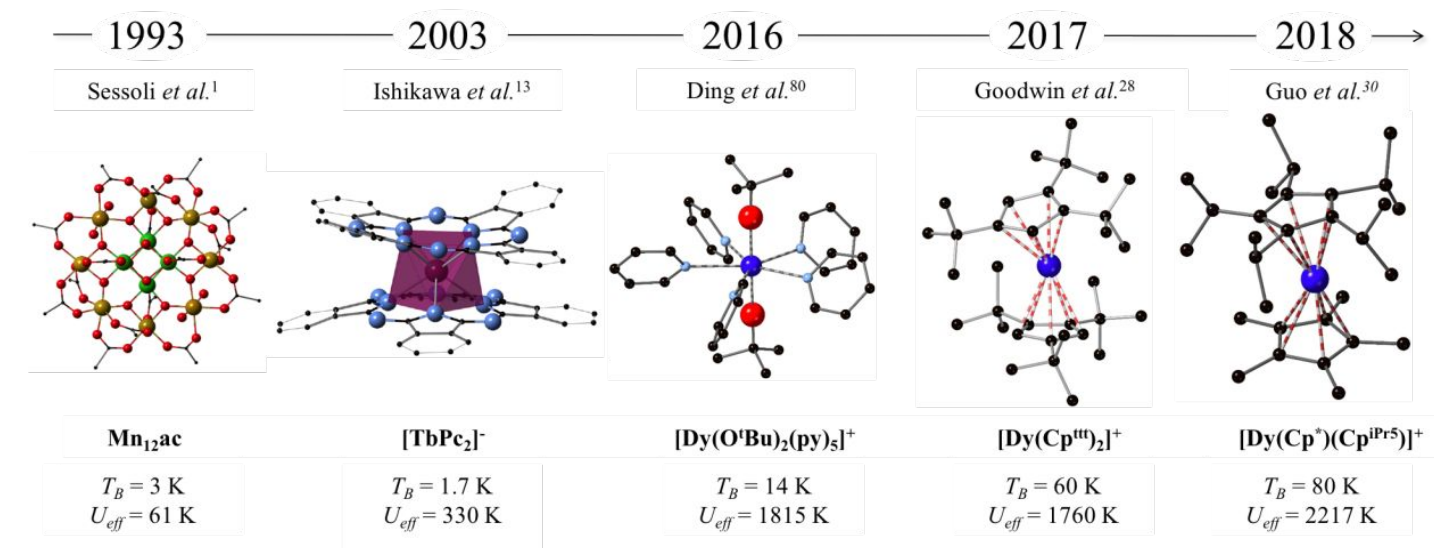

Figure 1. Milestones in molecular nanomagnets of rising values of effective barriers $U_{\text {eff }}$ and blocking temperatures $T_{B}$. 
Electrostatic crystal-field models aim to describe the perturbation of the $4 f$-shell electron cloud of the central ion produced by a surrounding charge distribution that breaks the degeneracy within the ground $J$ multiplet. The simplest model of this type is the Point Charge Electrostatic Model (PCEM), in which the electrostatic field is parameterized as a sum of Coulomb fields created by point charges (i.e. the formal charges) placed at the crystallographic positions, completely disregarding covalency. ${ }^{32}$ In molecular magnetism, this basic but intuitive idea permitted a preliminary attempt to rationalize the most likely conditions that lanthanoid-based complexes need to meet to behave as SIMs, both qualitatively ${ }^{31}$ and quantitatively. ${ }^{33,34}$ Based on the PCEM, the MAGELLAN code was developed as an inexpensive tool for the easy axis direction of low-symmetry dysprosium SIMs in $2013 .{ }^{35}$ Since the middle of last century, a plethora of modifications of this early model have been suggested in the literature. ${ }^{36,37,38,39,40}$ The idea has been to keep the simplicity of PCEM to some extent, but providing a more realistic set of crystal-field parameters (CFPs) as the spectroscopic techniques allowed a better estimation of them. Among those improvements, the Radial Effective Charge (REC) proposed by our group has often been used for the inexpensive modeling of molecular nanomagnets ${ }^{41}$ and spin qubits, ${ }^{42}$ The essential change of the REC model is the substitution of the formal charges of the atoms in the first coordination sphere by effective charges that are smaller than the real charges and are placed at smaller distances from the metal to account for covalency. For that, two parameters per type of donor atom are defined, namely the effective charge $\left(Z_{\text {eff }}\right)$ and the radial displacement $\left(D_{\mathrm{r}}\right)$. In a fitting procedure, both REC parameters are varied to achieve the minimum deviation between calculated and experimental data. The displacement of the point charge along the chemical bond direction has remarkable consequences in the values of the second-, fourth- and sixth-rank CFPs, correcting the deviations that had historically been encountered when comparing the PCEM-derived CFPs with phenomenological ones. ${ }^{43}$

On the other hand, recent advances in post Hartree-Fock multi-configurational $a b$ initio methodologies have permitted to develop a computational framework that has widely been used in the theoretical characterization of these magnetic entities. The main difference with regard to semiempirical or phenomenological methods is that first principles approaches do not rely on experimental data to be fitted. This means that their results are derived from the fundamental constants introduced by the applied quantum physical laws. Thus, it provides a perfect scenario to correlate the chemical structure with the magnetic and spectroscopic properties limited instead by computational time consumption. The most broadly employed $a b$ initio multi-configurational approach is the CASSCF/PT2 method (Complete Active Space Self-Consistent Field and Perturbation Theory up to Second Order), subsequently complemented with spin-orbit interaction effects (e.g. RASSI-SO, Restricted Active Space State Interaction with Spin-Orbit coupling). ${ }^{44,45,46}$ This method has been implemented in a plethora of computational packages such as MOLCAS -the most historically used in molecular magnetism-,${ }^{47,48}$ Gaussian, ${ }^{49}$ Orca $^{50}$ and MOLPRO $^{51}$. It allows the calculation of the full set of CFPs, energy levels, wave functions, as well as the orientation of the main magnetic axes and the static magnetic properties. The basics of this approach have deeply been described in the literature. Very recently, simplifications to CASSCF via the configuration-averaged Hartree-Fock (CAHF) algorithm with the objective of achieving similar results that those of CASSCF while reducing the time-scale of the calculations have been proposed..$^{52,53}$ The CASSCF/RASSI-SO method has played a determinant role in the understanding of the static magnetic properties of molecular nanomagnets and spin qubits. Through the SINGLE_ANISO program, it has been possible to compute magnetic anisotropy, ${ }^{54}$ magnetic susceptibility, magnetization, energy levels, magnetic relaxation pathways ${ }^{55}$ and magnetic couplings, ${ }^{56}$ as well as the full set of 27 CFPs. ${ }^{57}$ In this approach, CFPs are deduced by a decomposition of the CF matrix in irreducible tensor operators (ITOs) and in extended Stevens operators working in the ground ${ }^{2 S+1} L_{J}$ multiplet. Simultaneously, the projections of the total angular momentum with respect to the reference coordinate frame are also estimated. The first remarkable application of $a b$ initio methods to a mononuclear SMM dates back to 2012, when CASPT2 calculations were able to simulate the magnetic anisotropy changes of $\left[\mathrm{Na}\left\{\mathrm{Dy}(\mathrm{DOTA})\left(\mathrm{H}_{2} \mathrm{O}\right)\right\}\right] \cdot 4 \mathrm{H}_{2} \mathrm{O}$, derived by the rotation of the apical water molecule of the complex. ${ }^{58,59}$ Soon, this approach became very popular -one can find near a hundred of relevant results from Web of Science concerning mainly the application of CASSCF in $f$-element molecular magnetism-, and its success in the prediction of magnetic anisotropy, especially in Dy complexes, has been demonstrated again and again.

The following step in the rationalization and modeling of $f$-block molecular nanomagnets has been the first attempts towards the application of the above-mentioned theoretical frameworks developed for $3 \mathrm{~d}$ and $4 \mathrm{f}$ molecular nanomagnets to the study of actinoid-based SIMs. Unfortunately, this is not a straightforward procedure, as the more covalent character of the $5 \mathrm{f}$ orbitals adds more complexity to their theoretical description, thus hampering a rational design of these nanomagnets exhibiting improved properties. Due to the larger crystal field splitting that this type of complexes do possess, one needs to define a "full model" Hamiltonian taking into account inter-electronic repulsion, spin-orbit coupling and the ligand field potential. ${ }^{60}$ In this context, the CONDON package appears as the leading software code for this modeling. The best strategy is to provide an initial guess of the CFPs using either electrostatic or ab initio methods and then diagonalize the full Hamiltonian or fit a series of experimental energy levels by varying the calculated CFPs, as has recently been illustrated. ${ }^{61}$ In any case, one should note that in uranium, perhaps even more than with lanthanides, there is a large mismatch between $U_{\text {eff }}$ and energy gaps, of one to two orders of magnitude. ${ }^{62}$ This means that a simple Orbach process cannot describe the behavior properly and has stimulated some authors to assume a Raman process. Meihaus et al. showed that the temperature-dependent data of many $\mathrm{U}^{3+}$-SIMs might be fit well to a power dependence of temperature. ${ }^{63}$ This serves as 
a powerful stimulus for a more detailed modelling of the vibrationally-induced relaxation in actinides.

\section{Modeling the effect of vibrations on single-ion magnets}

One of the most recent crucial advances in this context, from the theoretical point of view, has been the incorporation of the effect of both molecular and lattice vibrations that couple to the spin states into the computational methodologies (see Fig. 2). Indeed, the quantitative improvement between $\left[\mathrm{Dy}\left(\mathrm{Cp}^{\mathrm{ttt}}\right)_{2}\right]^{+}\left(T_{B}=60\right.$ $\mathrm{K})^{28,29}$ and $\mathrm{Dy}-5^{*}\left(T_{B}=80 \mathrm{~K}\right)^{30}$ was justified as a rational optimization of the molecular structure in terms of vibrations. ${ }^{30}$ Since the hydrogen atoms in the $\mathrm{Cp}$ rings were involved in the vibrations that contributed most to the Orbach relaxation, these were substituted by aliphatic groups. This, of course, required a judicious design to find the exact steric impediment that allows coordination to the Dy of two substituted $\mathrm{Cp}$ rings while impeding a third ligand to join the coordination sphere. In this case, achieving this compromise while eliminating any hydrogen atom directly connected to the $\mathrm{Cp}$ rings meant overcoming the synthetic complication of combining two different rings, one with bulky isopropyl groups and another with the smaller methyl groups. Moreover, the theoretical analysis of Layfield and co-workers ${ }^{30}$ indicates that two out-of-plane vibrations of the $\mathrm{Cp}$ * ligand with frequencies in the range of the calculated gap to the first excited state (about $650 \mathrm{~cm}^{-1}$ ) might be responsible for relaxation in Dy-5*, thereby indicating the direction for further improvements: choosing substituents to bring these modes out of resonance with the excitation gap.

As in the case of the modeling of the ligand field, the modeling of the coupling between vibrations and spin energy levels also started with transition metals. ${ }^{64} \mathrm{~A}$ contribution by some of us introduced one of the germinal ideas on which current models rely on, ${ }^{65}$ already in 2015 , based on the case study of the square planar complex $\left[\mathrm{Cu}(\mathrm{mnt})_{2}\right]^{2-}\left(\mathrm{mnt}^{2-}=1,2\right.$-dicyanoethylene-1,2-dithiolate $)$. This was a systematic procedure to estimate the coupling between the electron spin energy levels and each individual molecular vibrational mode. The model relies on the ability to calculate, for any given geometry, the parameter $B$ in the Hamiltonian that governs spin energy levels, such as the effective Landé $g$ factor, the Zero Field Splitting $E$ or the CFPs $\left\{B_{2}^{0} \ldots B_{6}^{6}\right\}$. By preparing a set of molecular geometries along the distortion coordinate $Q_{k}$ of each vibrational mode $k$, by re-calculating this parameter $B$ for each of these coordinate sets, and by assuming a vibrational harmonic model, a Taylor expansion up to second order in mode coordinates leads to a simple expression for the coupling of $B$ with each mode $k$. This coupling is a function of the vibrational frequency of the mode, its reduced mass, and the second derivative of $B$ with respect to $Q_{k}$ evaluated at the relaxed geometry. It is important to note that this early model did not yet calculate relaxation times but merely matrix elements for the future master equations. 
TUTORIAL REVIEW

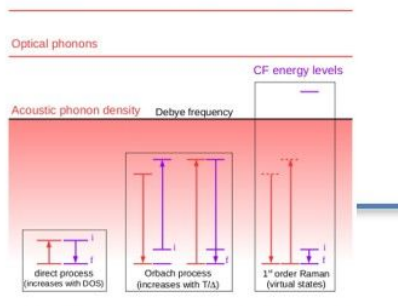

Liddle et al., Chem. Soc. Rev., 2015, 44, 6655-6669

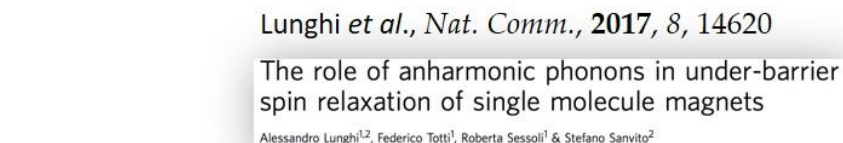

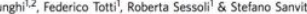

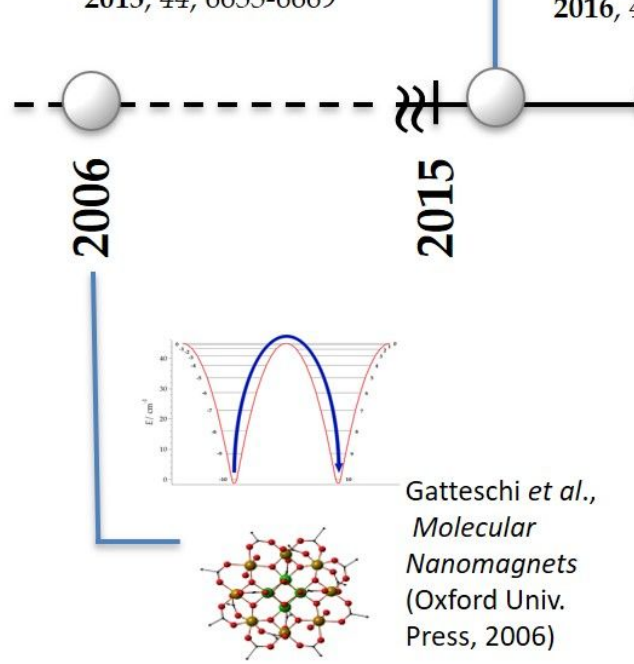

Reproduced from ref. 64. Copyright (2018, Royal Society of Chemistry).



(1)
(n)

Figure 2. Milestones in the theoretical modeling of vibration-mediated spin relaxation in molecular nanomagnets.

In 2017, a more sophisticated work was developed by Lunghi et al., ${ }^{66}$ based on the trigonal complex $[(\mathrm{tpaPh}) \mathrm{Fe}]^{-}$ (tpa=tris(pyrrolyl-R-methyl)amine). This contribution introduced a series of new key concepts into this research line, which allow to apply a correction to the Arrhenius plots and to explain why for high barrier cases the effective barrier can be much lower. Working under the Born-Markov approximation, the authors integrate out the phonons' component of the density matrix and reduce the problem to a purely electronic one in the presence of a phonon bath. The spin dynamics are then studied through the first-orderreduced spin density operator as described by the diagonal elements of the Redfield master equation, where phonon dissipation is explicitly introduced by modeling their spectral shape. Indeed, this model considers the phonon linewidth $\Delta$ that, after a threshold temperature, scales linearly with temperature. Importantly, this linewidth serves to explain the role of anharmonic phonons to achieve the resonance condition and ultimately the transfer of energy from an excited spin state towards the phonon thermal bath. Since the authors in this contribution solve the master equation, an estimate of the full relaxation time is calculated as a function of temperature. This work was accompanied by related advances by the same authors, which demonstrate the importance of local vibrational modes in magnetic relaxation, ${ }^{67}$ and correlate their harmonic frequencies with experiments from $\mathrm{THz}$ spectroscopy. ${ }^{68}$ Late progress on this line includes further first-principle spin dynamics by analyzing the role of coordination geometry and ligand field strength. ${ }^{69}$ In the case of vanadyl complexes, the relaxation mechanism is dominated, depending on whether the magnetic field is low or high, by the modulation of hyperfine coupling or of the Zeeman effect by the intra-molecular components of the acoustic phonons. ${ }^{70}$

After the ground was set by studies in transition metal complexes, the modeling of the coupling between vibrations and spin energy levels in lanthanide ions was finally kickstarted by the two recent records in high-temperature magnetic hysteresis, both based on the chemical family of the dysprosocenium sandwich. ${ }^{28-30}$ In both cases, the spinphonon coupling was determined by performing an extensive series of CASSCF-SO calculations for molecular geometries distorted along each vibrational mode. The variation of the CFPs was subsequently estimated from crystal field decomposition of the electronic structure for each of the distorted structures. One major approximation common to both works, namely to ignore long-wavelength phonons and to focus only on gas-phase local vibrations, may suppose an important limitation. A recent theoretical work provided preliminary insight into this open problem by finding -for a different lanthanide complex- that longwavelength phonons and low-frequency vibrations (below $10 \mathrm{~cm}^{-1}$ ) do not modulate significantly the spin energy levels in the solid state.

Crucially, Goodwin and co-workers ${ }^{28}$ went a step further and re-defined the vibrationally-induced Orbach and 
second-order Raman transition rates between two given electronic states. By assuming the Born-Oppenheimer approximation and a harmonic vibrational perturbation to the equilibrium electronic structure, the new expressions depend on a finite set of non-interacting harmonic vibrations as provided by first-principles geometry relaxations, instead of the common vibrational energy continuum derived from the use of the Debye model. These expressions allow building the so-called master matrix, whose diagonalization results in a series of characteristic relaxation rates that describe the several relaxation channels of the system. In particular, when the Orbach transition rates are employed, all but one are fast relaxation rates corresponding to processes at either side of the barrier, and the remaining slow relaxation rate corresponds to the Orbach relaxation over the barrier.

The next frontier in this challenging effort is the modeling of vibration-induced spin relaxation processes in actinide complexes. These systems combine the difficulties for the modeling of their electronic structure, as detailed in the previous section, with a scarcity in experimental results. Owing to the fact that the $5 \mathrm{f}^{3}$ electrons of $\mathrm{U}^{3+}$ may provide an oblate single-ion anisotropy, ${ }^{21}$ a particularly interesting family is that of uranocenium, which in principle could extend the promise of the hysteresis records experimentally found for dysprosocenium complexes under a rational design.

\section{The uranium case: differences in the design strategies for $\mathbf{4 f}$ and $5 f$ SIMs}

In this analysis we must bear in mind that the ultimate goal of first-principles calculations is the rational design of SIMs with advanced capabilities. This means, primarily, to enhance the low operating temperatures that still hamper their implementation in devices working at practical temperatures. Towards this goal, the modelling of the relaxation mechanisms that start to dominate demagnetization at these higher temperatures is of key importance. Indeed, as temperature is raised, the increasing energy stored in the phonon bath starts being sufficient to promote magnetic relaxation, as the spin makes use of this thermal energy and crosses the potential barrier that was impeding its reversal. This particular process is possible because of the so-called spin-vibration coupling. Thus, any attempt to rationalize and predict new SIMs operating at high temperatures needs to necessarily understand the interaction between spins and vibrations. In particular, current theoretical efforts are essentially aimed at identifying those molecular vibrations responsible for demagnetization. At the same time, such gain in knowledge is enabling the redesign of molecular ligands in order to suppress the relevant atomic movements that significantly couple to the spin and promote its relaxation, ${ }^{30}$ as described at the beginning of the previous section.

Given the potential for a larger ligand field splitting and enhanced covalence due to the greater extension of $5 \mathrm{f}$ orbitals, actinide-based SIMs have been proposed as interesting candidates for designing molecules with larger relaxation barriers than those of $4 \mathrm{f}$ SIMs. Nevertheless, either because of the number of 5f SIMs is still scarce or because of the lack of theoretical works focusing on the spin relaxation processes of these molecular entities, nowadays actinide-based are far to reach the performance of lanthanide-based molecular nanomagnets in terms of $U_{\text {eff }}$ and $T_{B}$. In this section, we will center our attention on $\mathrm{U}^{3+}-$ based SIMs, which are the most among $5 \mathrm{f}$ SIMs, but in contrast with those based on 4 f elements, are sufficiently few to be comprehensively reviewed in some detail.

The first reported example of a mononuclear uranium coordination complex exhibiting slow relaxation of magnetization was reported by the Long group a decade ago, namely $\mathrm{U}\left(\mathrm{Ph}_{2} \mathrm{BPz}_{2}\right)_{3} \quad(\mathbf{1}),{ }^{21}$ where $\left[\mathrm{Ph}_{2} \mathrm{BPz}_{2}\right]^{-}=$ diphenylbis(pyrazolyl)borate (Fig. 3). A year later, the two phenyl groups were replaced by hydrogen atoms, obtaining the second uranium-based SIM, $\mathrm{U}\left(\mathrm{H}_{2} \mathrm{BPz}_{2}\right)_{3}$ (2). ${ }^{71}$ Although both derivatives possess a very similar trigonal prismatic coordination environment with $\sim D_{3 h}$ symmetry, noticeable differences concerning $U_{\text {eff }}$ were obtained $\left(U_{\text {eff }}(\mathbf{1})=28.8 \mathrm{~K}\right.$ and $\left.U_{\text {eff }}(2)=11.5 \mathrm{~K}\right)$. Interestingly, the latter showed an additional relaxation process which could be effectively suppressed by diamagnetic dilution, reaching an energy barrier of $23 \mathrm{~cm}^{-1}$ in $\mathrm{U}_{0.01} \mathrm{Y}_{0.99}\left(\mathrm{Ph}_{2} \mathrm{BPz}_{2}\right)_{3}{ }^{72}$ In parallel, two new uranium-based molecular nanomagnets, namely $\left[\mathrm{U}\left(\mathrm{Tp}^{\mathrm{Me} 2}\right)_{2}(\text { bipy })\right]^{+}$(3) and its precursor $\mathrm{U}\left(\mathrm{Tp}^{\mathrm{Me} 2}\right)_{2} \mathrm{I}$ (4), based on $\mathrm{Tp}^{\mathrm{Me} 2}=$ hydrotris(3,5-dimethylpyrazolyl)borate ligands, were synthesized and characterized by Almeida's group. ${ }^{73,26}$ The effective energy barriers of $\mathbf{3}(26.2 \mathrm{~K})$ and 4 $(30.3 \mathrm{~K})$ were quantitatively similar to those of $\mathbf{1}$, which contrasted with the almost an order of magnitude larger energy gaps calculated using the $a b$ initio SO-CASPT2 method (198.6 K and 210.1 K for 3 and 4, respectively). In fact, the fifth-reported $\mathrm{U}^{3+} \operatorname{SIM}\left(\mathrm{UTp}_{3}(5), \mathrm{Tp}^{-}=\right.$ trispyrazolylborate) evidenced more dramatically such large difference between the ground to first excited state energy gap $(388.5 \mathrm{~K})$, in this case determined by luminescence, ${ }^{74}$ and $U_{\text {eff }}(5.5 \mathrm{~K}) \cdot{ }^{75}$ The static magnetic properties of these five complexes were rationalized by means of the REC model by some of us, providing then an estimation of their energy levels and ground state wave functions together with a first analysis of which geometries are more suitable to obtain uranium-based SIMs. ${ }^{23}$ In 2013, Liddle and van Slageren and co-workers reported three $\mathrm{U}^{3+}$ complexes, $\left[\mathrm{UI}_{3}(\mathrm{THF})_{4}\right](6)(\mathrm{THF}=$ tetrahydrofuran $), \mathrm{U}\left[\mathrm{N}\left(\mathrm{SiMe}_{3}\right)_{2}\right]$ (7) and $\left(\mathrm{U}\left(\mathrm{CH}\left(\mathrm{PPh}_{2} \mathrm{NSiMe}_{3}\right)_{2}\right](\mathrm{I})_{2}(\mathrm{THF})(\mathbf{8})\right)$, displaying slow relaxation of the magnetization, with $U_{\text {eff }}$ (coordination symmetry) of $18.6 \mathrm{~K}\left(\sim C_{2 v}\right), 29 \mathrm{~K}\left(\sim C_{3 v}\right)$ and $23.4 \mathrm{~K}\left(C_{1}\right)$ for 6, 7 and 8, respectively. ${ }^{22}$ Subsequently, the impact of the donor strength was evaluated in two isostructural trigonal prismatic compounds, $\mathrm{U}\left(\mathrm{Bp}^{\mathrm{Me}}\right)_{3}$ (9) and $\mathrm{U}\left(\mathrm{Bc}^{\mathrm{Me}}\right)_{3}$ (10), where $\left[\mathrm{Bp}^{\mathrm{Me}}\right]^{-}=$dihydrobis(methypyrazolyl)borate and $\left[\mathrm{Bc}^{\mathrm{Me}}\right]^{-}=$dihydrobis(methylimidazolyl)borate). While 9 did not show SMM behavior, the diluted analogue of $\mathbf{1 0}$ exhibited $U_{\text {eff }}=47.6 \mathrm{~K}$, which is the largest documented for a $\mathrm{U}^{3+}$ SIM (see Fig. 3). ${ }^{76}$ This different behavior was attributed to the greater magnetic anisotropy imposed by the $\mathrm{N}$-heterocyclic carbene coordination sphere. The most recent examples to be added to the list are a trigonal prismatic SIM with general formula 
$\left[\mathrm{U}\left\{\mathrm{SiMe}_{2} \mathrm{NPh}\right\}_{3}\right.$-tacn $\left.)\left(\mathrm{OPPh}_{3}\right)\right] \quad(\mathbf{1 1}) \quad\left(U_{\text {eff }}=21.9 \mathrm{~K}\right)$, $\operatorname{tacn}=1,4,7$-triazacyclononane, which has been investigated by a combination of thermodynamic and spectroscopic techniques, together with $a b$ initio calculations that have been used as a starting point for the phenomenological description, ${ }^{61}$ and the uranocenium cationic complex of general formula $\left[\mathrm{U}\left(\eta^{5}-\mathrm{C}_{5}{ }^{\mathrm{iPr} 5}\right)_{2}\right]^{+}(\mathbf{1 2}),{ }^{77}$ formed by two cyclopentadienyl ligands (Fig. 4d). This latter system is of special interest due to the groundbreaking results in terms of $U_{\text {eff }}$ and $T_{B}$ that $\mathrm{Dy}^{3+}$-based metallocenes have achieved in the last few years. Almost simultaneously, we have recently provided the first theoretical effort to explore the spinvibrational coupling in a foreseeable uranocenium SIM -the (hypothetical) uranium analogue of $\left[\mathrm{Dy}\left(\mathrm{Cp}^{\mathrm{ttt}}\right)_{2}\right]^{+}$(Figure $\left.4 \mathrm{a}\right)$ namely $\left[\mathrm{U}\left(\mathrm{Cp}^{\mathrm{ttt}}\right)_{2}\right]^{+}$(Figure 4b)-. ${ }^{78}$ These calculations exploit the parameterization of the crystal field effect of $\left[\mathrm{Cp}^{\mathrm{tt} t}\right]^{-}$ligands within the REC model, so that the effect of the ligand is reproduced by modelling each donor atom by a radially displaced effective electric charge that thereafter is applied to the uranium analogue. ${ }^{78,41}$



Figure 3. Molecular structures of a selection of uranium-based SIMs and their respective $U_{\text {eff }}$.

In spite of the limited available data compared with lanthanide-based SIMs, one can find some common patterns regarding wave function purity, symmetry and expected effects of vibrational distortions in the reported $5 \mathrm{f}^{3}$ uranium SIMs, so they suffice to offer some early insights. Let us start by the composition of their wave functions. It is noteworthy that every uranium complex analyzed in Ref. 23 presents a ground doublet characterized by a very strong mixing between $M_{J}$ microstates, whether this is within the same doublet and/or with an excited state. These are also the cases of the ground doublets of $\mathbf{1 0}$ and 11, where EPR measurements suggest ground states characterized by a mixture of $M_{J}= \pm 5 / 2$ and $M_{J}= \pm 7 / 2$ in both of them. Even in uranocenium, the calculated ground doublet shows a considerable mixing of $M_{J}= \pm 9 / 2, M_{J}= \pm 5 / 2$ and $M_{J}=$ $\pm 3 / 2$. $^{78}$ This common pattern seems to be not at all an accidental consequence of particular molecular geometries, and rather a general feature caused by the low total $J=9 / 2$ of $\mathrm{U}^{3+}$ and the importance of the fourth-order CFPs in $\mathrm{U}^{3+}$ complexes. In fact, in theory the highest $M_{J}$ in the ground doublet could be either $M_{J}= \pm 9 / 2$ or $M_{J}= \pm 7 / 2$, but they are in all the investigated cases mixed. In particular, and this does depend on molecular geometry, in the reported examples so far, there are important $\pm M_{J}=3$ mixings in the low-lying levels. For example, if there is a $M_{J}= \pm 9 / 2$ weight in the ground doublet, there is also at least some $M_{J}= \pm 3 / 2$ either in the ground doublet or in the first excited doublet; similarly, if there is a $M_{J}= \pm 7 / 2$ weight in the ground doublet, there is also at least some $M_{J}= \pm 1 / 2$ either in the ground doublet or in the first excited doublet.

Of course, this means that any variation in a rank 3 extra-diagonal CFP effects remarkable changes in the mixing and can thus be relevant to facilitate fast spin relaxation. Indeed, it seems that extra-diagonal CFPs of rank 3 , such as $B_{4}^{3}$, are especially relevant for the majority of the existing uranium SIMs, both from the point of view of the molecular symmetries and of the compositions of the ground state. This fact needs to be considered for chemical design efforts, since $D_{3 h}$ (or near- $D_{3 h}$ ) is a very common symmetry for uranium compounds, and if the coordination is close to $D_{3 h}$, all vibrations that are antisymmetric with respect to $\sigma_{h}\left(\mathrm{~A}_{1}{ }^{\prime}\right.$, $\mathrm{A}_{2}$ " and E" in Mulliken's notation) are expected to contribute to massive changes in rank 3 extra-diagonal CFPs. Interestingly, even in the case of metallocenium complexes, where the ligands impose a symmetry that could be seen as a strongly distorted $D_{5 d}$, theoretical analysis of the coupling between vibrations and crystal field parameters in $\left[\mathrm{Dy}\left(\mathrm{Cp}^{\mathrm{tt} t}\right)_{2}\right]^{+}$and Dy-5* indicates that $B_{4}^{3}$ also suffers very strong coupling with vibrations, much more than $B_{6}^{5}$ which naïvely would be more adequate from the point of view of symmetry. ${ }^{28,79}$ A similar behavior is predicted for $\left[\mathrm{U}\left(\mathrm{Cp}^{\mathrm{ttt}}\right)_{2}\right]^{+}$, where the coupling $B_{4}^{3}$ is predicted to present a very intense relative thermal dependence. ${ }^{78}$

Thus, the combination of heavily mixed wave functions and the coupling of such mixing to vibrations that are expected for symmetry reasons, might offer an explanation to the somewhat disappointing results that have been achieved so far in uranium SIMs. Note that all the reported nanomagnets show $U_{\text {eff }} \sim 20-30 \mathrm{~K}$ with $47.6 \mathrm{~K}$ as a record energy barrier ${ }^{76}$ (Fig. 3) and magnetic hysteresis below $5 \mathrm{~K}$ in the best of the cases. ${ }^{26}$ This contrasts with the obtained $U_{\text {eff }}$ for some dysprosium complexes, e.g. [Dy $\left.\left(\mathrm{Cp}^{\mathrm{ttt}}\right)_{2}\right]^{+}\left(U_{\text {eff }}\right.$ $=1760 \mathrm{~K}),{ }^{28,29} \mathrm{Dy}-5^{*}\left(U_{\text {eff }}=2217 \mathrm{~K}\right)^{30}$ or $\left[\mathrm{Dy}\left(\mathrm{O}^{\mathrm{t} B u}\right)_{2}(\mathrm{py})_{5}\right]^{+}$ $\left(U_{\text {eff }}=1815 \mathrm{~K}\right)^{80}$, and magnetic hysteresis up to $80 \mathrm{~K},{ }^{30}$ which might illustrate the need to set up different design strategies for $5 \mathrm{f}$ SIMs.

\section{Exploring the metallocenium path}

A more promising approach within uranium-based molecular nanomagnets, which will need to be deeply explored in the following years, is the uranocenium family (Figure 4b-4d). Very recently, the first example (12) has been prepared by abstracting iodide from $\left[\mathrm{U}\left(\mathrm{Cp}^{\mathrm{iPr} 5}\right)_{2} \mathrm{I}\right](\mathbf{1 3})$. Surprisingly, slow relaxation of the magnetization could only be observed after applying an external magnetic field of 1 and $1.5 \mathrm{KOe}$ for 12 and 13, respectively, which evidences the presence of efficient quantum tunneling of the magnetization. This is compatible with the reported CASSCF $a b$ initio calculations that result in an energy gap between the ground and excited doublets of $\sim 300 \mathrm{~cm}^{-1}$, but significant transverse contributions to the $g$ tensors. This behavior is strikingly different to that of its $\mathrm{Dy}^{3+}$ analogue, namely $\left[\mathrm{Dy}\left(\mathrm{Cp}^{\mathrm{iPr} 5}\right)_{2}\right]^{+}$(Figure $\left.4 \mathrm{c}\right)$, which displays an $U_{\text {eff }}=$ $2112.1 \mathrm{~K}$ and magnetic hysteresis up to $72 \mathrm{~K}$ at a sweep rate of $3.1 \mathrm{mTs}^{-1}{ }^{81}$ Since uranium is bulkier than dysprosium, it 
could be expected that a larger degree of steric hindrance would be needed to achieve an equivalent linearity. However, a geometrical analysis of their chemical structures reveals an angle $\alpha(\mathrm{Cp}-\mathrm{U}-\mathrm{Cp})=167.8^{\circ}$ for $\left[\mathrm{U}\left(\mathrm{Cp}^{\mathrm{iPr} 5}\right)_{2}\right]^{+}$that deviates significantly less from linearity than the original $\left[\mathrm{Dy}\left(\mathrm{Cp}^{\mathrm{iPr} 5}\right)_{2}\right]^{+}$, with possess an angle $\alpha(\mathrm{Cp}-\mathrm{Dy}-\mathrm{Cp})$ of $162.1^{\circ}$. As discussed above, the problem with uranium is not achieving linearity but rather the heavy mixing in the ground doublet, even for very linear environments, and its sensibility to certain extra-diagonal terms that are expected to couple strongly with common vibrations. This fact is a clear limitation of these complexes with respect to those of the second half of lanthanide series, for example, $\mathrm{Tb}^{3+}-$, $\mathrm{Dy}^{3+}-, \mathrm{Ho}^{3+}$ - or $\mathrm{Er}^{3+}$-based SIMs. a)



c)

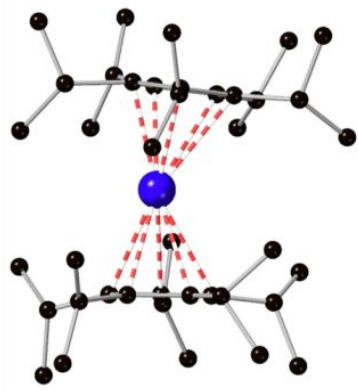

b)



d)



Figure 4. Molecular structures of (a) $\left[\mathrm{Dy}\left(\mathrm{Cp}^{\mathrm{ttt}}\right)_{2}\right]^{+}$, (b) $\left[\mathrm{U}\left(\mathrm{Cp}^{\mathrm{ttt}}\right)_{2}\right]^{+}$, (c) $\left[\mathrm{Dy}\left(\mathrm{Cp}^{\mathrm{iPr} 5}\right)_{2}\right]^{+}$, (d) $\left[\mathrm{U}\left(\mathrm{Cp}^{\mathrm{iPr} 5}\right)_{2}\right]^{+}$. Hidrogen atoms have been omitted for clarity.

A similar comparison can be done between $\left[\mathrm{Dy}\left(\mathrm{Cp}^{\mathrm{ttt}}\right)_{2}\right]^{+}$ (Figure 4a), where we have access to both experimental and theoretical results, and the theoretical estimates in $\left[\mathrm{U}\left(\mathrm{Cp}^{\mathrm{ttt}}\right)_{2}\right]^{+}$(Figure 4b). In this case there is a larger deviation from linearity, in both cases, but especially in the uranium case: $\quad \alpha(\mathrm{Cp}-\mathrm{U}-\mathrm{Cp})=142.4^{\circ} \quad$ vs $\quad \alpha(\mathrm{Cp}-\mathrm{Dy}-$ $\mathrm{Cp})=152.6^{\circ}$. This results in a less axial environment and a much more compressed energy level scheme for $\left[\mathrm{U}\left(\mathrm{Cp}^{\mathrm{ttt}}\right)_{2}\right]^{+}$, with heavy mixing, as can be observed in Ref. 78. An analysis of the equilibrium electronic structure in these two cases reveals the composition of the spin states in terms of the $\left|M_{J}\right\rangle$ labels corresponding to the ground $J$ multiplet. In $\left[\mathrm{U}\left(\mathrm{Cp}^{\mathrm{ttt}}\right)_{2}\right]^{+}$, the ground doublet is composed in a $82 \%$ of $| \pm 9 / 2\rangle$, being the $\mid M_{J}>$ mixing even more noticeable in the excited doublets. As discussed above, this extensive mixing coincides with what has also been calculated in the previously reported uranium-based nanomagnets, ${ }^{61}$ and is assumed to facilitate magnetic relaxation. Furthermore, calculations showed that in $\left[\mathrm{U}\left(\mathrm{Cp}^{\mathrm{ttt}}\right)_{2}\right]^{+}$the spin tunnels the barrier just a bit above the first excited doublet. ${ }^{78}$ These features are in sharp contrast with those exhibited by the dysprosocenium magnets $\left[\mathrm{Dy}\left(\mathrm{Cp}^{\mathrm{ttt}}\right)_{2}\right]^{+}$(and Dy-5*), where (i) the low-lying spin states are much purer although the ligand coordination is not strictly axial, and (ii) the barrier tunneling is produced close to the most excited doublets. ${ }^{28-}$ ${ }^{30}$ Thus, according with this electronic structure scheme, the SMM performance of $\left[\mathrm{U}\left(\mathrm{Cp}^{\mathrm{ttt}}\right)_{2}\right]^{+}$is expected to be worse than of dysprosocenium magnets. To quantify this conclusion the timescale of the relaxation pathway also needs to be considered. Estimated relaxation times in $\left[\mathrm{U}\left(\mathrm{Cp}^{\mathrm{ttt}}\right)_{2}\right]^{+}$, with expected hysteresis temperatures up to 10 $\mathrm{K}$, are clearly much shorter than those reported for $\left[\mathrm{Dy}\left(\mathrm{Cp}^{\mathrm{ttt}}\right)_{2}\right]^{+}$and Dy-5*, presenting hysteresis up to $60 \mathrm{~K}$ and $80 \mathrm{~K}$ respectively. The fact that the calculated relaxation is much higher than the experimental characterization of $\left[\mathrm{U}\left(\mathrm{Cp}^{\mathrm{iPr} 5}\right)_{2}\right]^{+}$, where no hysteresis is reported and what is observed at $10 \mathrm{~K}$ is the start of the $a c$ out-of-phase signal at $1 \mathrm{kHz}$, should be taken with a grain of salt. Indeed, this theoretical methodology is expected to estimate an upper bound to relaxation times, since it necessarily disregards many relaxation paths, including all vibrational effects beyond the single molecule, e.g. extended phonons. ${ }^{82,83}$

Finally, another intriguing aspect to consider concerns one of the key steps in the design of a long magnetic memory in the original dysprosocenium, which was the elimination of the equatorial ligand $\mathrm{I}^{-}$to achieve a uniaxial coordination. In contrast, Layfield and co-workers have reported minimal differences in the relaxation of $\left[\mathrm{U}\left(\mathrm{Cp}^{\mathrm{iPr} 5}\right)_{2}\right]^{+}$compared to that of $\left[\mathrm{U}\left(\mathrm{Cp}^{\mathrm{iPr} 5}\right)_{2} \mathrm{I}\right] .{ }^{77}$ The reason behind this shocking difference can probably be found in the very different electronic structures of $\mathrm{Dy}^{3+}$ complexes with respect to $\mathrm{U}^{3+}$ complexes. Whereas $\mathrm{Dy}^{3+}$ complexes have the potential for an extremely well defined $M_{J}= \pm 15 / 2$ doublet, uranium complexes tend to present important mixing in the ground states. $^{23,84}$ Again, the ground states of $\left[\mathrm{U}\left(\mathrm{Cp}^{\mathrm{iPr} 5}\right)_{2}\right]^{+}$and $\left[\mathrm{U}\left(\mathrm{Cp}^{\mathrm{iPr} 5}\right)_{2} \mathrm{I}\right]$ are likely to be similarly non-pure, as we have theoretically estimated in the analogous case of $\left[\mathrm{U}\left(\mathrm{Cp}^{\mathrm{ttt}}\right)_{2}\right]^{+} .^{78}$ In this case, the relative success of the metallocenium strategy, both experimentally in the cases of $\left[\mathrm{U}\left(\mathrm{Cp}^{\mathrm{iPr} 5}\right)_{2}\right]^{+}$and $\left[\mathrm{U}\left(\mathrm{Cp}^{\mathrm{iPr} 5}\right)_{2} \mathrm{I}\right]$ and theoretically in the case of $\left[\mathrm{U}\left(\mathrm{Cp}^{\mathrm{ttt}}\right)_{2}\right]^{+}$is necessarily based on the rigidity of the $\mathrm{Cp}$ ligands that tend to reduce vibrationally-induced spin relaxation.

\section{Conclusions}

Molecular nanomagnets based on rare-earth complexes have been breaking records of performance. This has been possible by following a design strategy based on highly axial, highly rigid metallocenium-type complexes. Further structural structural exploration of metallocenium-type structures is likely to yield even better results. Whereas this strategy is being extremely successful in dysprosium-based molecular nanomagnets, reaching unprecedented blocking temperatures up to $80 \mathrm{~K}$, the first reported uranocenium does not even show slow relaxation of the magnetization in zero field and the theoretical predictions for $\left[\mathrm{U}\left(\mathrm{Cp}^{\mathrm{ttt}}\right)_{2}\right]^{+}$are far from outperforming the properties of the lanthanoid analogues. The revision of theoretical modeling of different kinds of SIMs and of the few existing examples of uranium 
SIMs evidences the need of a better understanding of the role of vibrations in uranium spin dynamics. Despite the initial promise of actinide ions, which can present very strong crystal field effects and thus large expected effective barriers, the behavior of the uranium SIMs reported so far is poor compared with the best dysprosium-based molecular nanomagnets. This is undoubtedly due to the intense mixing in the ground doublet in uranium complexes that allows efficient quantum tunneling of the magnetization, even for coordination environments that are rather axial, but also to the stronger coupling of uranium's spin energy levels with molecular vibrations. Uranium-based SIMs seem to require their own rational design, since simple transmutation strategies, where the actinoid ion is substituted for dysprosium in a carefully designed $\mathrm{Dy}^{3+}$ SIM might not be enough to exploit its full potential.

\section{ASSOCIATED CONTENT}

\section{AUTHOR INFORMATION}

\section{Corresponding Author}

*E-mail for E.C.: eugenio.coronado@uv.es

*E-mail for J.J.B.: jose.baldovi@mpsd.mpg.de

\section{ACKNOWLEDGMENT}

The present work has been funded by the EU (COST Action CA15128 MOLSPIN and ERC-2014-CoG-647301 DECRESIM), the Spanish MINECO (Unit of Excellence "María de Maeztu" MdM-2015-0538, and grants MAT2017-89993-R and CTQ2017-89528-P) and the Generalitat Valenciana (Prometeo Program of Excellence). EU-QUANTERA project SUMO is also acknowledged. L. E.-M. also thanks the Generalitat Valenciana for a VALi+D predoctoral contract, J. J. B. acknowledges the EU for a Marie Curie Fellowship (H2020-MSCA-IF-2016-751047).

\section{ABBREVIATIONS}

SIM, single-ion magnet; SMM, single-molecule magnet; PCEM, point-charge electrostatic model; CFPs, crystal-field parameters; REC, Radial Effective Charge; CASSCF, Complete Active Space Self-Consistent Field; CASPT2, Complete Active Space Perturbation Theory up to Second Order; SO, spin-orbit; Cp, cyclopentadienyl.

\section{REFERENCES}

\footnotetext{
${ }^{1}$ Sessoli, R.; Gatteschi, D.; Caneschi, A.; Novak, M. A. "Magnetic bistability in a metal-ion cluster." Nature 1993, 365, $141-143$.

2 Thomas, L.; Lionti, F.; Ballou, R.; Gatteschi, D.; Sessoli, R.; Barbara, B. "Macroscopic quantum tunnelling of magnetization in a single crystal of nanomagnets." Nature 1996, 383, 145-147.

${ }^{3}$ Bagai, R.; Christou, G. "The drosophila of single-molecule magnetism:[Mn12O12(O2CR)16(H2O)4] ." Chem. Soc. Rev. 2009, 38, 10111026.

${ }^{4}$ Bertaina, S.; Gambarelli, S.; Mitra, T.; Tsukerblat, B.; Müller, A.; Barbara, B. "Quantum oscillations in a molecular magnet.” Nature 2008, 453, 203-206.

${ }^{5}$ Leuenberger, M. N.; Loss, D. "Quantum computing in molecular magnets." Nature 2001, 410, 789-793.

${ }^{6}$ Troiani, F.; Affronte, M. "Molecular spins for quantum information technologies." Chem. Soc. Rev. 2011, 40(6), 3119-3129.

7 Bogani, L.; Wernsdorfer, W. "Molecular spintronics using single-molecule magnets." Nanoscience And Technology: A Collection of Reviews from Nature Journals. 2010, 7, 179-186.

${ }^{8}$ Gaita-Ariño, A.; Luis, F.; Hill, S.; Coronado, E. "Molecular spins for quantum computation." Nat. Chem. 2019, 11(4), 301-309.

${ }^{9}$ Waldmann, O. "A criterion for the anisotropy barrier in single-molecule magnets." Inorg. Chem. 2007, 46, $10035-10037$.

${ }^{10}$ Chakov, N. E.; Lee, S.- C.; Harter, A. G.; Kuhns, P. L.; Reyes, A. P.; Hill, S.; Dalal, N. S.; Wernsdorfer, W.; Abboud, K. A.; Christou, G. "The Properties of the [Mn12O12(O2CR)16(H2O)4] Single-Molecule Magnets in Truly Axial Symmetry:[Mn12O12(O2CCH2Br)16 (H2O)4]·4CH2C12." J. Am. Chem. Soc. 2006, 128, 6975-6989.

${ }^{11}$ Milios, C. J.; Vinslava, A.; Wernsdorfer, W.; Moggach, S.; Parsons, S.; Perlepes, S. P.; Christou, G.; Brechin, E. K. "A record anisotropy barrier for a single-molecule magnet." J. Am. Chem. Soc., 2007, 129, 2754-2755.

${ }^{12}$ Chakarawet, K.; Bunting, P. C.; Long, J. R. "Large anisotropy barrier in a tetranuclear single-molecule magnet featuring low-coordinate cobalt centers." J. Am. Chem. Soc. 2018, 140, 2058-2061.

${ }^{13}$ Ishikawa, N.; Sugita, M.; Ishikawa, T.; Koshihara, S.; Kaizu, Y. "Lanthanide double-decker complexes functioning as magnets at the single-molecular level." J. Am. Chem. Soc., 2003, 125, 8694-8695.

${ }^{14}$ Meng, Y.-S.; Jiang, S.-D.; Wang, B.-W.; Gao, S. "Understanding the magnetic anisotropy toward single-ion magnets." Acc. Chem. Res. 2016, 49(11), 2381-2389.

${ }^{15}$ Woodruff, D.N.; Winpenny, R.E.P.; Layfield, R. A. "Lanthanide single-molecule magnets." Chem. Rev. 2013, 113(7), 5110-5148.

${ }^{16}$ Craig, G.A.; Murrie, M. "3d single-ion magnets." Chem. Soc. Rev. 2015, 44, 2135-2147.

${ }^{17}$ Feng, M.; Tong, M. L. "Single ion magnets from 3d to 5f: Developments and strategies." Chem. Eur. J., 2018, 24, 7574-7594.

18 Zadrozny, J. M.; Xiao, D. J.; Atanasov, M.; Long, G. J.; Grandjean, F.; Neese, F.; Long, J. R. "Magnetic blocking in a linear iron (I) complex." Nat. Chem. 2013, 5, 577-581.

${ }^{19}$ Yao, X.-N.; Du, J.-Z.; Zhang, Y.-Q.; Leng, X.-B.; Yang, M.-W.; Jiang, S.-D.; Wang, Z.-X.; Ouyang, Z.-W.; Deng, L.; Wang, B.-W.; Gao, S. "Two-coordinate Co (II) imido complexes as outstanding single-molecule magnets." J. Am. Chem. Soc., 2017, 139, 373-380..

${ }^{20}$ Bunting, P. C.; Atanasov, M.; Damgaard-Møller, E.; Perfetti, M.; Crassee, I.; Orlita, M.; Overgaard, J.; van Slageren, J.; Neese, F.; Long, J. R. “A linear cobalt (II) complex with maximal orbital angular momentum from a non-Aufbau ground state.” Science, 2018, 362(6421),
} 
eaat7319.

${ }^{21}$ Rinehart, J. D.; Long, J. R. "Slow magnetic relaxation in a trigonal prismatic uranium (III) complex." J. Am. Chem. Soc. 2009, 131, 1255812559.

${ }^{22}$ Moro, F.; Mills, D. P.; Liddle, S. T.; van Slageren, J. "The inherent single-molecule magnet character of trivalent uranium." Angew. Chem. Int. Ed. 2013, 52(12), 3430-3433.

${ }^{23}$ Baldoví, J. J.; Cardona-Serra, S.; Clemente-Juan, J. M.; Coronado, E.; Gaita-Ariño, A. "Modeling the properties of uranium-based single ion magnets." Chem. Sci. 2013, 4, 938-946.

${ }^{24}$ Antunes, M. A.; Coutinho, J. T.; Santos, I. C.; Marçalo, J.; Almeida, M.; Baldoví, J. J.; Pereira, L. C. J.; Gaita-Ariño, A.; Coronado, E. "A mononuclear uranium (IV) single-molecule magnet with an azobenzene radical ligand." Chem.-Eur. J. 2015, 21(49), 17817-17826.

${ }^{25}$ Magnani, N.; Apostolidis, C.; Morgenstern, A.; Colineau, E.; Griveau, J.-C.; Bolvin, H.; Walter, O.; Caciuffo, R. "Magnetic memory effect in a transuranic mononuclear complex." Angew. Chem., Int. Ed., 2011, 50, 1696-1698.

${ }^{26}$ Coutinho, J. T.; Antunes, M. A.; Pereira, L. C. J.; Bolvin, H.; Marçalo, J.; Mazzanti, M.; Almeida, M. "Single-ion magnet behaviour in [U(TpMe2)2I]." Dalton Trans. 2012, 41, 13568-13571.

${ }^{27}$ Harriman, K. L. M.; Murugesu, M. "An organolanthanide building block approach to single-molecule magnets." Acc. Chem. Res. 2016, $49,1158-1167$.

${ }^{28}$ Goodwin, C.A.P.; Ortu, F.; Reta, D.; Chilton, N.F. "Molecular magnetic hysteresis at 60 kelvin in dysprosocenium." Nature 2017, 548, 439-442.

${ }^{29}$ Guo, F. S.; Day, B. M.; Chen, Y. C.; Tong, M. L.; Mansikkamäki, A.; Layfield, R. A. “A Dysprosium Metallocene Single-Molecule Magnet Functioning at the Axial Limit" Angew. Chem. Int. Ed. 2017, 56, 11445-11449.

${ }^{30}$ Guo, F. S.; Day, B. M.; Chen, Y. C.; Tong, M. L.; Mansikkamäki, A.; Layfield, R. A. "Magnetic hysteresis up to 80 kelvin in a dysprosium metallocene single-molecule magnet." Science 2018, 362(6421), 1400-1403.

${ }^{31}$ Rinehart, J. D.; Long, J. R. "Exploiting single-ion anisotropy in the design of f-element single-molecule magnets." Chem. Sci. 2011, 2 , 2078-2085.

32 Bethe, H. A. "Splitting of terms in crystals." Ann. Phys. 1929, 3, 133-206.

${ }^{33}$ Baldoví, J. J.; Cardona-Serra, S.; Clemente-Juan, J. M.; Coronado, E.; Gaita-Ariño, A.; Palii, A. "Rational design of single-ion magnets and spin qubits based on mononuclear lanthanoid complexes." Inorg. Chem. 2012, 51, 12565-12574.

${ }^{34}$ Baldoví, J. J.; Cardona-Serra, S.; Clemente-Juan, J. M.; Coronado, E.; Gaita-Ariño, A.; Palii, A. "SIMPRE: A software package to calculate crystal field parameters, energy levels, and magnetic properties on mononuclear lanthanoid complexes based on charge distributions." $J$. Comput. Chem. 2013, 34, 1961-1967.

${ }^{35}$ Chilton, N. F.; Collison, D.; McInnes, E. J.; Winpenny, R. E.; Soncini, A. "An electrostatic model for the determination of magnetic anisotropy in dysprosium complexes." Nat. Commun. 2013, 4, 2551.

${ }^{36}$ Jorgensen, C. K.; Pappalardo, R.; Schmidtke, H.-H. "Do the "Ligand Field" Parameters in Lanthanides Represent Weak Covalent Bonding?." J. Chem. Phys. 1963, 39, 1422-1430.

${ }^{37}$ Urland, W. "On the ligand-field potential for f electrons in the angular overlap model." Chem. Phys. 1976, 14, 393-401.

${ }^{38}$ Malta, O. L. "A simple overlap model in lanthanide crystal-field theory." Chem. Phys. Lett. 1982, 87, 27-29.

${ }^{39}$ Malta, O. L. "Theoretical crystal-field parameters for the YOCl: Eu3+ system. A simple overlap model" Chem. Phys. Lett. 1982, 88, 353356.

${ }^{40}$ Porcher, P.; Couto Dos Santos, M.; Malta, O. L. "Relationship between phenomenological crystal field parameters and the crystal structure: The simple overlap model." Phys. Chem. Chem. Phys. 1999, 1, 397-405.

${ }^{41}$ Baldoví, J. J.; Borrás-Almenar, J. J.; Clemente-Juan, J. M.; Coronado, E.; Gaita-Ariño, A. "Modeling the properties of lanthanoid singleion magnets using an effective point-charge approach." Dalton Trans. 2012, 41, 13705-13710.

${ }^{42}$ Baldoví, J. J.; Rosaleny, L. E.; Ramachandran, V.; Christian, J.; Dalal, N. S.; Clemente-Juan, J. M.; Yang, P.; Kortz, U.; Gaita-Ariño, A.; Coronado, E. "Molecular spin qubits based on lanthanide ions encapsulated in cubic polyoxopalladates: design criteria to enhance quantum coherence." Inorg. Chem. Front. 2015, 2, 893-897.

${ }^{43}$ Baldoví, J. J.; Gaita-Ariño, A.; Coronado, E. "Modeling the magnetic properties of lanthanide complexes: relationship of the REC parameters with Pauling electronegativity and coordination number." Dalton Trans. 2015, 44, 12535-12538.

${ }^{44}$ Roos, B. O.; Taylor, P. R.; Sigbahn, P. E. M. "A complete active space SCF method (CASSCF) using a density matrix formulated superCI approach." Chem. Phys. 1980, 48, 157-173.

${ }^{45}$ Siegbahn, P.; Heiberg, A.; Roos, B.; Levy, B. "A comparison of the super-CI and the Newton-Raphson scheme in the complete active space SCF method." Phys. Scr. 1980, 21, 323-327.

${ }^{46}$ Siegbahn, P. E. M.; Almlöf, J.; Heiberg, A.; Roos, B. O. "The complete active space SCF (CASSCF) method in a Newton-Raphson formulation with application to the HNO molecule." J. Chem. Phys. 1981, 74, 2384-2396.

${ }^{47}$ Aquilante, F.; De Vico, L.; Ferré, N.; Ghigo, G.; Malmqvist, P. Å.; Neogrády, P.; Pedersen, T. B.; Pitoňák, M.; Reiher, M.; Roos, B. O.; Serrano-Andrés, L.; Urban, M.; Veryazov, V.; Lindh, R. "MOLCAS 7: the next generation." J. Comput. Chem., $2010,31,224-247$.

${ }^{48}$ Karlström, G.; Lindh, R.; Malmqvist, P. Å.; Roos, B. O.; Ryde, U.; Veryazov, V.; Widmark, P. O.; Cossi, M.; Schimmelpfennig, B.; 
Neogrady, P.; Seijo, L. "MOLCAS: a program package for computational chemistry." Comp. Mat. Sci. 2003, 28, $222-239$.

${ }^{49}$ Frisch, M. J.; Trucks, G. W.; Schlegel, H. B.; Scuseria, G. E.; Robb, M. A.; Cheeseman, J. R.; Scalmani, G.; Barone, V.; Mennucci, B.; Petersson, G. A.; Nakatsuji, H.; Caricato, M.; Li, X.; Hratchian, H. P.; Izmaylov, A. F.; Bloino, J.; Zheng, G.; Sonnenberg, J. L.; Hada, M.; Ehara, M.; Toyota, K.; Fukuda, R.; Hasegawa, J.; Ishida, M.; Nakajima, T.; Honda, Y.; Kitao, O.; Nakai, H.; Vreven, T.; Montgomery, J. A.; Peralta, J. E., Jr.; Ogliaro, F.; Bearpark, M.; Heyd, J. J.; Brothers, E.; Kudin, K. N.; Staroverov, V. N.; Kobayashi, R.; Normand, J.; Raghavachari, K.; Rendell, A.; Burant, J. C.; Iyengar, S. S.; Tomasi, J.; Cossi, M.; Rega, N.; Millam, J. M.; Klene, M.; Knox, J. E.; Cross, J. B.; Bakken, V.; Adamo, C.; Jaramillo, J.; Gomperts, R.; Stratmann, R. E.; Yazyev, O.; Austin, A. J.; Cammi, R.; Pomelli, C.; Ochterski, J. W.; Martin, R. L.; Morokuma, K.; Zakrzewski, V. G.; Voth, G. A.; Salvador, P.; Dannenberg, J. J.; Dapprich, S.; Daniels, A. D.; Farkas, O.; Foresman, J. B.; Ortiz, J. V.; Cioslowski, J.; Fox, D. J. "Gaussian 09, Revision A” Gaussian, Inc., Wallingford CT, 2009.

${ }^{50}$ Neese F. "The ORCA program system." Wiley Interdiscip. Rev. Comput. Mol. Sci., 2012, 2, 73-78.

${ }^{51}$ Werner, H. J.; Knowles, P. J.; Knizia, G.; Manby, F. R.; Schütz, M. "Molpro: a general-purpose quantum chemistry program package." Wiley Interdiscip. Rev. Comput. Mol. Sci., 2012, 2, 242-253.

${ }^{52}$ Hallmen, P. P.; Köppl, C.; Rauhut, G.; Stoll, H.; van Slageren, J. "Fast and reliable ab initio calculation of crystal field splittings in lanthanide complexes." J. Chem. Phys., 2017, 147, 164101.

${ }^{53}$ Calvello, S.; Piccardo, M.; Rao, S. V.; Soncini, A. "CERES: An ab initio code dedicated to the calculation of the electronic structure and magnetic properties of lanthanide complexes." J. Comput. Chem. 2018, 39(6), 328-337.

${ }^{54}$ Ungur, L.; Chibotaru, L. F. "Magnetic anisotropy in the excited states of low symmetry lanthanide complexes." Phys. Chem. Chem. Phys., 2011, 13, 20086- 20090.

${ }^{55}$ Blagg, R. J.; Ungur, L.; Tuna, F.; Speak, J.; Comar, P.; Collison, D.; Wernsdorfer, W.; McInnes, E. J. L.; Chibotaru, L. F.; Winpenny, R. E. P. "Magnetic relaxation pathways in lanthanide single-molecule magnets." Nat. Chem. 2013, 5, 673-678.

${ }^{56}$ Guo, Y. N.; Xu, G. F.; Wernsdorfer, W.; Ungur, L.; Guo, Y.; Tang, J.; Zhang, H. J.; Chibotaru, L. F.; Powell, A.K. "Strong axiality and ising exchange interaction suppress zero-field tunneling of magnetization of an asymmetric Dy2 single-molecule magnet." J. Am. Chem. Soc. 2011, 133, 11948-11951.

${ }^{57}$ Chibotaru, L. F.; Ungur, L. "Ab initio calculation of anisotropic magnetic properties of complexes. I. Unique definition of pseudospin Hamiltonians and their derivation." J. Chem. Phys. 2012, 137(6), 064112.

${ }^{58}$ Cucinotta, G.; Perfetti, M.; Luzón, J.; Etienne, M.; Car, P. E.; Caneschi, A.; Calvez, G.; Bernot, K.; Sessoli, R. "Magnetic anisotropy in a dysprosium/DOTA single-molecule magnet: beyond simple magneto-structural correlations." Angew. Chem. Int. Ed., 2012, 51, 1606-1610.

${ }^{59}$ Boulon, M. E.; Cucinotta, G.; Luzón, J.; Degl'Innocenti, C.; Perfetti, M.; Bernot, K.; Calvez, G.; Caneschi, A.; Sessoli, R. "Magnetic Anisotropy and Spin-Parity Effect Along the Series of Lanthanide Complexes with DOTA." Angew. Chem. Int. Ed., 2013, 52, 350-354.

${ }^{60}$ Speldrich, M.; van Leusen, J; Kögerler, P. "CONDON 3.0: An Updated Software Package for Magnetochemical Analysis-All the Way to Polynuclear Actinide Complexes." J. Comput. Chem. 2018, 39, 2133-2145.

${ }^{61}$ Coutinho, J. T.; Perfetti, M.; Baldoví, J. J.; Antunes, M. A.; Hallmen, P. P.; Bamberger, H.; Crassee, I.; Orlita, M.; Almeida, M.; van Slageren, J.; Pereira, L. C. "Spectroscopic Determination of the Electronic Structure of a Uranium Single-Ion Magnet." Chem.-A Eur. J., 2019, 25(7), 1758-1766.

${ }^{62}$ McAdams, S. G.; Ariciu, A. M.; Kostopoulos, A. K.; Walsh, J. P.; Tuna, F. "Molecular single-ion magnets based on lanthanides and actinides: Design considerations and new advances in the context of quantum technologies." Coord. Chem. Rev. 2017, 346, $216-239$.

${ }^{63}$ Meihaus, K.R.; Long, J.R. "Actinide-based single-molecule magnets." Dalton Trans. 2015, 44(6), $2517-2528$.

${ }^{64}$ Escalera-Moreno, L.; Baldoví, J. J.; Gaita-Ariño, A.; Coronado, E. "Spin states, vibrations and spin relaxation in molecular nanomagnets and spin qubits: a critical perspective." Chem. Sci., 2018, 9, 3265-3275.

65 (a) Escalera-Moreno, L.; Suaud, N.; Gaita-Ariño, A.; Coronado, E. "Role of vibrations on decoherence in molecular spin qubits: The case of [Cu(mnt)2]2-." 2015, arXiv:1512.05690v1 (b) Escalera-Moreno, L.; Suaud, N.; Gaita-Ariño, A.; Coronado, E. "Determining key local vibrations in the relaxation of molecular spin qubits and single-molecule magnets." J. Phys. Chem. Lett. 2017, 8, 1695-1700.

${ }^{66}$ Lunghi, A.; Totti, F.; Sessoli, R.; Sanvito, S. "The role of anharmonic phonons in under-barrier spin relaxation of single molecule magnets." Nat. Commun. 2017, 8, 14620.

${ }^{67}$ Lunghi, A.; Totti, F.; Sanvito, S.; Sessoli, S. "Intra-molecular origin of the spin-phonon coupling in slow-relaxing molecular magnets." Chem. Sci., 2017, 8, 6051-6059.

${ }^{68}$ Atzori, M.; Tesi, L.; Benci, S.; Lunghi, A.; Righini, R.; Taschin, A.; Torre, R.; Sorace, L.; Sessoli, R. "Spin dynamics and low energy vibrations: insights from vanadyl-based potential molecular qubits." J. Am. Chem. Soc., 2017, 139, 4338-4341.

${ }^{69}$ Albino, A.; Benci, S.; Tesi, L.; Atzori, M.; Torre, R.; Sanvito, S.; Sessoli, R.; Lunghi, A. "First-principles investigation of spin-phonon coupling in vanadium-based molecular spin qubits." 2019, arXiv:1904.04922.

${ }^{70}$ Lunghi, A.; Sanvito, S. "Spin-Phonon Relaxation in Molecular Qubits from First Principles Spin Dynamics." 2019, arXiv:1903:01424.

${ }^{71}$ Rinehart, J. D.; Meihaus, K. R.; Long, J. R. "Observation of a secondary slow relaxation process for the field-induced single-molecule magnet U(H2BPz2)3.” J. Am. Chem. Soc., 2010, 132(22), 7572-7573.

${ }^{72}$ Meihaus, K. R.; Rinehart, J. D.; Long, J. R. "Dilution-induced slow magnetic relaxation and anomalous hysteresis in trigonal prismatic dysprosium (III) and uranium (III) complexes." Inorg. Chem. 2011, 50(17), 8484-8489. 
${ }^{73}$ Antunes, M. A.; Pereira, L. C.; Santos, I. C.; Mazzanti, M.; Marçalo, J.; Almeida, M. "[U(TpMe2)2(bipy)]+: A Cationic Uranium (III) Complex with Single-Molecule-Magnet Behavior.” Inorg. Chem., 2011, 50(20), 9915-9917.

${ }^{74}$ Apostolidis, C.; Morgenstern, A.; Rebizant, J.; Kanellakopulos, B.; Walter, O.; Powietzka, B.; Karbowiak, M.; Reddmann, H.; Amberger, H.-D. Z. Anorg. Allg. Chem. 2010, 636, 201.

${ }^{75}$ Rinehart, J. D.; Long, J. R. "Slow magnetic relaxation in homoleptic trispyrazolylborate complexes of neodymium (III) and uranium (III)." Dalton Trans., 2012, 41(44), 13572-13574.

${ }^{76}$ Meihaus, K. R.; Minasian, S. G.; Lukens Jr, W. W.; Kozimor, S. A.; Shuh, D. K.; Tyliszczak, T.; Long, J. R. "Influence of pyrazolate vs N-heterocyclic carbene ligands on the slow magnetic relaxation of homoleptic trischelate lanthanide (III) and uranium (III) complexes." $J$. Am. Chem. Soc., 2014, 136(16), 6056-6068.

${ }^{77}$ Layfield, R.; Guo, F. S.; Mansikkamaki, A.; Tong, M. L.; Chen, Y. C. "Uranocenium: synthesis, structure and chemical bonding." Angew. Chem. 2019, https://doi.org/10.1002/ange.201903681.

${ }^{78}$ Escalera-Moreno, L.; Baldoví, J. J.; Coronado, E. "An efficient general methodology to explore the high-temperature frontier in f-block molecular nanomagnets" arXiv:1905.06989

${ }^{79}$ Ullah, A. "Molecular engineering of dysprosocenium: towards minimum spin-phonon coupling and maximum magnetic memory", work in preparation.

${ }^{80}$ Ding, Y. S.; Chilton, N. F.; Winpenny, R. E. P.; Zheng, Y. Z. "On approaching the limit of molecular magnetic anisotropy: a near-perfect pentagonal bipyramidal dysprosium (III) single-molecule magnet." Angew. Chem. Int. Ed., 2016. 55(52), $16071-16074$.

${ }^{81}$ McClain, K. R.; Gould, C. A.; Chakarawet, K.; Teat, S. J.; Groshens, T. J.; Long, J. R.; Harvey, B. G. "High-temperature magnetic blocking and magneto-structural correlations in a series of dysprosium (III) metallocenium single-molecule magnets." Chem. Sci., 2018, 9(45), 8492-8503.

${ }^{82}$ Hizhnyakov, V.; Pae, K.; Vaikjarv, T. "Optical Jahn-Teller Effect in the Case of Local Modes and Phonons." Chem. Phys. Lett. 2012, 525-526, 64-68.

${ }^{83}$ Palii, A.; Ostrovsky, S.; Reu, O.; Tsukerblat, B.; Decurtins, S.; Liu, S.-X.; Klokishner, S. "Diversity of Spin Crossover Transitions in Binuclear Compounds: Simulation by Microscopic Vibronic Approach” J. Phys. Chem. C, 2016, 120(26), 14444-14453.

${ }^{84}$ Magnani, N. "Spectroscopic and magnetic investigations of actinide-based nanomagnets." Int. J. Quant. Chem., 2014, 114(12), 755-759. 
We offer a brief perspective of the progress on the theoretical modelling of single-ion magnetism and focus on the open problem of understanding and optimizing the vibrationally-induced spin relaxation in uranium SIM complexes, including an experimental and a theoretical study of the promising metallocenium family. 